\title{
Fire Modeling: Where Are We? Where Are We Going?
}

\author{
KEVIN McGRATTAN \\ Building and Fire Research Laboratory \\ National Institute of Standards and Technology \\ Gaithersburg, Maryland, USA
}

\begin{abstract}
Current trends in computational fluid dynamics (CFD) modeling of fire are discussed, in particular the widening communication gap between model developers, model users, and the larger fire protection engineering community in regard to combustion and turbulence. The paper suggests steps that can be taken by all of these groups to improve the current situation and move forward to develop better fire models.
\end{abstract}

KEYWORDS: computational fluid dynamics (CFD), modeling

\section{INTRODUCTION}

Last year, I visited the Large Fire Lab at NIST to observe an experiment recreating the initial stages of the fire that killed 100 people in a Rhode Island nightclub in February, 2003 [1]. One of the aims of the experiment was to test our fire model, the Fire Dynamics Simulator (FDS), which was being used in the NIST investigation of the tragedy. I asked one of the technicians who was setting up the experiment, Lauren DeLauter, whether or not he expected to see flames emerge from a door at the rear of the test compartment. He said no, because the polyurethane foam lining the walls would burn rapidly, the compartment would become under-ventilated, and the unburned fuel gases would cool before reaching the exit. Because the mock-up was not designed to duplicate the geometry of the entire club, but rather the area in the immediate vicinity of the stage, his assessment did not contradict what was observed in the Rhode Island fire. Indeed, he was correct, whereas our model was wrong, in that it predicted flames emerging from the exit.

Although it appears to many that numerical simulation is the cutting edge of fire protection engineering, many non-modelers are surprised to learn that our ability to reproduce fire phenomena via computer simulation lags our empirical understanding by about 10 years. Indeed, current generation zone and field (computational fluid dynamics or CFD) models address transport phenomena reasonably well, making them useful for many engineering applications. For example, FDS did successfully replicate many of the phenomena associated with the Rhode Island nightclub fire. However, it has not yet reached the point of reliably predicting, for large scale applications, such important phenomena as flame spread, extinction, suppression, and CO and smoke production, all of which demand more detailed chemistry and physics than are currently incorporated in the model.

Hindering our efforts to move forward is the increasing level of miscommunication between modelers and experimentalists, scientists and engineers, and even professors and students. For various reasons, those who actually write the computer programs too often cannot or will not explain which algorithms work, which do not, and which have no effect at all on the results. This communication gap leads to unwarranted claims by end users who believe the models are predicting more than they actually are. Moving ahead will require that we bridge these gaps, so that all sectors of the fire and combustion 
communities can improve our theoretical understanding of fire behavior that we have all observed in the laboratory.

\section{WHERE ARE WE NOW?}

In the past few years, the US Nuclear Regulatory Commission (NRC) has sponsored an international working group to evaluate fire models for nuclear power plant applications. "Round Robins" of this sort have been held in the past by other groups for other reasons, but they all have a similar purpose. Participants simulate fire experiments and then assess the results of the models. In the NRC study, a series of increasingly complex fire tests have been conducted at various laboratories, with the intent of pushing the limits of the models. The models consist of hand calculations, zone models and CFD models. One broad conclusion has been that for simple geometries, all the models do a fairly good job in describing the bulk mass and energy transport. That is, upper and lower layer temperatures are predicted well enough for design purposes. CFD models have an advantage when the geometry is complex, and the simpler models have an advantage when a quick answer is needed, or hundreds of quick answers are needed for a probabilistic analysis. But some might say that this is where we were 10 years ago. Perhaps, but some changes have been made over the past decade to move us forward. Computers are faster and the models have become more robust and easier to use, and some progress has been made in modeling under-ventilated compartments and the burning of complicated targets, like power cables. However, the NRC study clearly identified these subjects as worthy of further development.

At this point, three different CFD techniques have emerged for modeling fire: Direct Numerical Simulation (DNS), Large Eddy Simulation (LES), and Reynolds-Averaged Navier-Stokes (RANS). DNS is really nothing more than a direct numerical solution of the governing equations. Because this technique demands very fine spatial and temporal resolution (about $1 \mathrm{~mm}$ or less), it is limited to small laminar flames and sometimes small turbulent jets. DNS is still not practical for large-scale fire simulations. LES and RANS employ models of the unresolved sub-grid dissipative processes. These "turbulence models" vary in scope, with LES attempting to compute as much of the "resolvable" length and time scales (the "large eddies") as possible (Fig. 1), whereas RANS averages over significantly larger spatial and temporal scales than those that are characteristic of the given numerical grid or the fundamental frequency of the fire.

While most accept the practical limitations of DNS as a fact of life, the debate over RANS and LES as applied to fire will continue. At times it seems like an argument over religion, but we're presently seeking middle ground. Attempts are currently underway to better explain these techniques, as well as their strengths and weaknesses. While we continue to debate which techniques are appropriate for various applications, we must stop saying that one method is "right" while another is "wrong". This is like saying that Rembrandt was a better painter than Picasso. Consider the three famous self-portraits in Fig. 2. Each of the three artists chose to represent reality in his own way, and the patrons of these artists obviously shared their vision. Wealthy European merchants of the 17th Century demanded portraits of the highest fidelity, literally what they saw in the mirror absent a few blemishes here and there. As photography developed in the late 19th Century, there was no longer such a demand for photo-realistic portraits, and artists began to explore different degrees of abstraction and interpretation. Although it's a bit of a stretch, DNS is like a Rembrandt portrait, whose level of detail is as fine as the brush the artist chose to use. Van Gogh and Picasso differ in the degree of abstraction each 
brought to the image. Van Gogh used a fairly large brush and thick strokes of paint to create images that were life-like only when observed at a distance. Picasso did not concern himself much with realism, especially in his later years. However, he he had the ability to capture the essence of something with just a few strokes of a brush or a piece of charcoal.

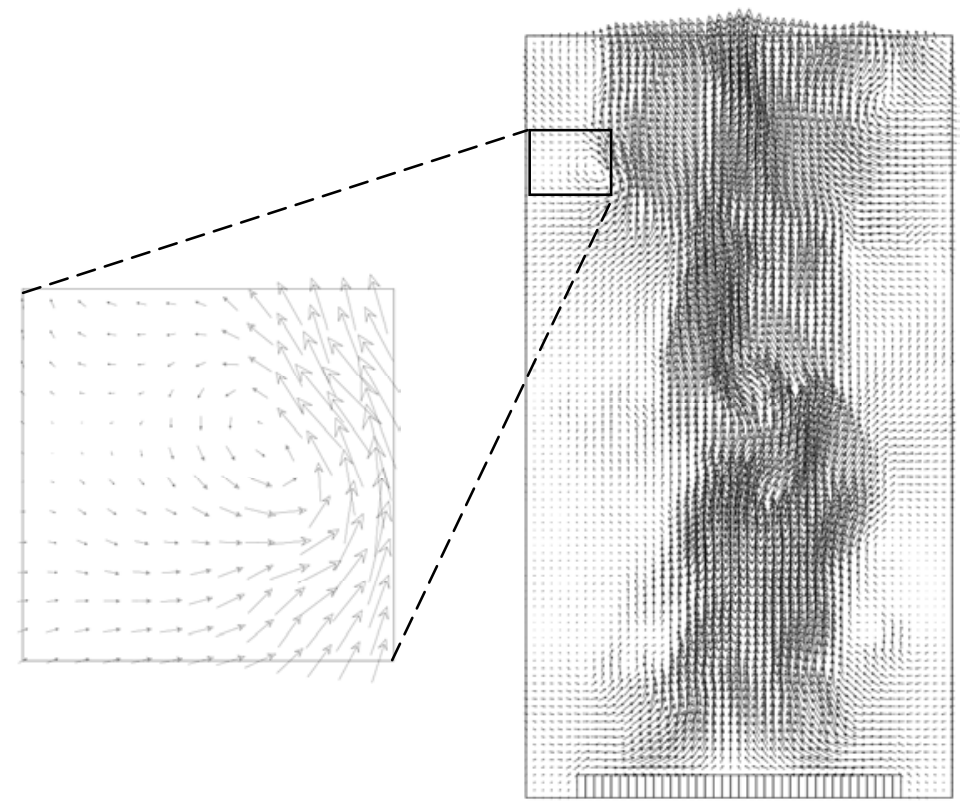

Fig. 1. What we mean by "resolvable." At right is an instantaneous map of the flow vectors for an LES simulation of a pool fire. The inset shows the smallest "eddy" that can be supported by the numerical grid, the spacing of which is indicated by the distance between the arrows.

At this point, I risk widening the turbulence debate beyond fire and combustion, so we return to the topic at hand. Many in the fire research community assert that the arguments over LES and RANS could be settled once and for all by having each type of model make "blind" predictions of the outcome of a given experiment. Trouble is, the "winner" would probably be scenario dependent. If for example, we all modeled the filling of a large hall with smoke, zone models or even hand calculations would provide answers as accurate or perhaps more accurate than the most sophisticated of CFD models because in some sense the "right" answer is built directly into the plume or ceiling jet correlations implicit in the methods. Many industrial processes - especially those for which we are only interested in some form of "steady-state" solution and whose turbulence can be characterized as "homogeneous" - would be handled well by RANS models, which is why most commercial, general purpose CFD models are of the RANS variety. At NIST, my colleagues Howard Baum and Ronald Rehm have always maintained that organized structures in fire plumes, ceiling jets, and other fire-driven flows are best represented with LES techniques, and we have demonstrated that these techniques are practical by developing FDS and validating it for a variety of common fire scenarios. We believe that as we develop more sophisticated models of the combustion processes within the fire itself, there will be no alternative to LES because the assumption that the fire can be time-averaged to the degree demanded by RANS models will not allow for a better 
description of the fire dynamics that we have all observed. This is our opinion, and it should not be misinterpreted to mean that we think RANS models are "wrong." There are several RANS models that have been developed specifically for fire, including JASMINE, KAMELEON, SMARTFIRE and SOFIE, plus various research and special application codes, which have all been shown to work well for a variety of fire scenarios. While the model developers continue to argue the merits of each technique, the model users should decide for themselves which is more appropriate for their particular applications. A useful survey of current fire models, including references, has been compiled by Olenick and Carpenter [2].
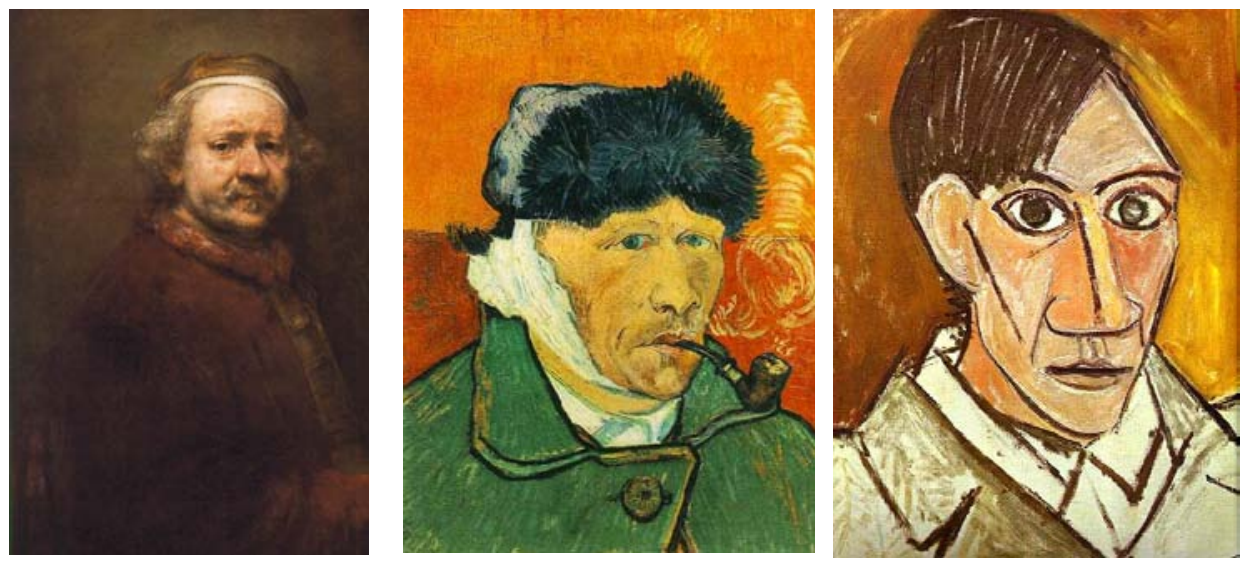

Fig. 2. Self-portraits of Rembrandt (Dutch, 1606-1669), Van Gogh (Dutch, 1853-1890) and Picasso (Spanish, 1881-1973). Note Van Gogh's formative work in Large Eddy Simulation.

\section{WHERE ARE WE GOING?}

Regardless of model type - zone, RANS, LES - we have fairly high confidence in our ability to model transport phenomena. In other words, if we are given the fire's heat release rate and the yields of the various exhaust products, we can predict compartment temperatures to within $20 \%$ at worst, and at best to within experimental accuracy. Which merely means that we can solve the mass, momentum and energy conservation equations reliably if we are given the source terms and boundary conditions. What's next? Obviously the source terms and boundary conditions, by which we really mean gas phase combustion and solid phase pyrolysis. In this paper, we will address the gas phase phenomena, not because the solid phase is less complicated, but because there exists in the literature a set of pyrolysis models of increasing complexity from which to choose, depending on the application and the desired degree of empiricism [3]. As for the gas phase, there is at present a wide gap between the simple "mixed is burnt" models, and the detailed kinetic mechanisms that are described in the combustion literature. For wellventilated compartment fires it is sufficient for most practical simulations to simply predict the transport of fuel and oxygen and burn them as they mix. We might argue about the best way to transport and mix the gases, but by and large, we're all making the assumption that the fuel and oxygen react readily on contact. However, the next step to take us beyond our simple combustion models appears to be a sizable leap into the unknown. 
I believe that combustion algorithms in fire models should focus on the carbon balance. Experimental work in the 1980's and 1990's has led us all to a fairly good understanding of the role played by soot, $\mathrm{CO}$, and unburned hydrocarbons in fire. The relatively simple experiments of Beyler [4], Zukoski [5], Pitts [6], and others [7] identified general trends that I believe can be explained to some extent in terms of the resolvable quantities, with a modest amount of empirical modeling. Currently, our models cannot predict the outcome of these experiments, except in certain circumstances. Note the emphasis on the word predict. We can track soot and CO reasonably well, and we often use empirical rules relating the global equivalence ratio and $\mathrm{CO} /$ soot generation, but we still can't make reliable predictions in large-scale, under-ventilated compartment fire simulations.

This is not because we don't know the chemical mechanisms. Numerous combustion theorists and modelers have written down detailed descriptions of them that would be more than adequate for our purpose if we could only extract from our coarse grids the necessary information needed by the detailed models. We refer to this process as "subgrid modeling," and it is tantalizing because it offers the hope of incorporating 100 years of combustion research into our fire models. But the dilemma that most have not faced (since they aren't burdened with actually implementing these sub-grid models in a working CFD model) is that roughly two orders of magnitude separate the large scales, say a grid cell, from the sub-grid scales, say the width of a flame. For example, a decent simulation of a house fire can be performed using grid cells on the order of $10 \mathrm{~cm}$. But the details of the flame - its temperature and local strain rate - require resolution down to at most $1 \mathrm{~mm}, 2$ orders of magnitude away. Even if we have information from, say, a counterflow diffusion experiment, what information can be passed from the $10 \mathrm{~cm}$ grid cell to the sub-grid model that will yield credible information? Combustion researchers are busily studying counterflow flames, and fire modelers are busily burning houses down, but who is bridging the gap?

Many in the combustion community maintain that we can predict detailed combustion phenomena now. For example, many point to flamelet libraries as a means to incorporate detailed chemistry into our fire models. A flamelet library is a vast collection of results of either small-scale diffusion flame experiments or calculations, conducted with various strain rates, oxygen/fuel concentrations, temperatures, fuel species, etc., and bundled up into an extensive "look-up table." A recent paper in Fire Safety Journal [8] reports 30,000 flamelet sets have been incorporated in the fire model SOFIE. We must ask what role the CFD fire model is playing in the simulations, that is, what information is being passed from the resolvable flow field to the flamelet library. In the FSJ paper, the oxygen content of the air entrained into the fire was the primary means of selecting one of the 30,000 flamelet sets. Given the complexity of the chemistry in this case, it is not unreasonable to invoke experimental results, but it must be made clear what information is being transferred from the CFD model to the flamelet library, and what information is being passed back. Current CFD fire models, like SOFIE, can predict the overall degree of vitiation within a compartment. The question remains, however, about the quality of the information that a CFD model can provide locally, relative to the detail inherent in the sub-grid model. No matter how good the flamelet library, the weak link is the information being passed from the resolvable scales.

Before we turn to very elaborate sub-grid models, we should reconsider the information that can be extracted directly from the resolvable scales. Are we exploiting this information as well as we can? Or are we trying to infer too much from it? For example, consider the simple mixture fraction combustion model used in FDS. It predicts with a 
single scalar transport equation the location of a surface at which fuel and oxygen meet at the proper stoichiometric ratio for burning, and it predicts the mass flux of fuel and oxygen across this surface. This is really the only prediction the model makes. What comes next can be considered sub-grid modeling. We assume that the local heat release rate is proportional to the mass flux of oxygen across the "flame sheet," and this energy is distributed to the grid cells through which the sheet passes. It is difficult to say, based on the resolvable information, whether or not the combustion is actually occurring, and if it is, how much of the energy heats up the gas and how much is emitted in the form of thermal radiation. Over the years, we have tried, in various ways, to use only the resolvable information to predict local extinction and radiative emission. However, we compromised the accuracy of the model trying to predict local extinction and radiative emission. Given only an "average" temperature and strain rate on our relatively coarse grids, we were confident only in assuming the local heat release rate from the oxygen flux, and prescribing the radiative loss as a fixed fraction of the local HRR. Using the resolved temperature field to predict the radiative loss produced inconsistent results because of its fourth power dependence on temperature.

We do not claim that our simple combustion model is the answer, and we continue to explore new methods. However, for any new method we consider, we must be clear about what information is being passed from the resolvable scales to the sub-grid model, and what is predicted and what is prescribed. Is the detail inherent in the sub-grid model commensurate with what is actually resolved by the large scale flow calculation? If we are to make progress on the carbon balance, I do not believe that the answer is in more detailed sub-grid models. Although they are a boon for chemists, flamelet libraries have more than enough detail already, and often deceive the user into believing that the fire model is doing more than it really is. It is important to look at what information our large scale calculations provide us - bulk temperatures, and simple combinations of $\mathrm{C}, \mathrm{O}, \mathrm{N}$ and $\mathrm{H}$. Before looking at $\mathrm{HCN}$ or $\mathrm{NO}_{\mathrm{x}}$ production, can we simply model the conversion of $\mathrm{CO}$ to $\mathrm{CO}_{2}$ in the hot upper layer of a room fire? Suppose we simplify the chemistry to something tractable at the resolvable scales [9]:

$$
\begin{aligned}
& \mathrm{C}_{\mathrm{n}} \mathrm{H}_{\mathrm{m}}+\left(\frac{\mathrm{n}}{2}+\frac{\mathrm{m}}{4}\right) \mathrm{O}_{2} \rightarrow \mathrm{nCO}+\frac{\mathrm{m}}{2} \mathrm{H}_{2} \mathrm{O} \\
& \mathrm{CO}+\frac{1}{2} \mathrm{O}_{2} \rightarrow \mathrm{CO}_{2}
\end{aligned}
$$

Shouldn't we start here before looking at more detailed models. I am not the first to suggest a simple 2-step mechanism, but for some reason we just have not explored it fully, choosing instead to pursue much more detailed chemistry. Can the hundreds of reactants and reactions bundled into the flamelet library tell us something that is more reliable than the information passed to it?

Consider what information we need from the large scale calculation. How many transport equations have to be solved to get us this information? A single mixture fraction variable will not do, as that implies that there is only one reaction, and it occurs instantaneously where fuel and oxygen meet at stoichiometric proportions. If we allow for the possibility that fuel and oxygen can mix and not burn, we need another scalar variable to keep track of the fraction of unburned fuel in the exhaust products. That's two transport equations 
now instead of one. Now, consider the $\mathrm{CO}$ produced by an under-ventilated fire mixing in the hot upper layer with entrained oxygen. Suppose we let it react and produce $\mathrm{CO}_{2}$. Now a third scalar is needed to keep track of the $\mathrm{CO} / \mathrm{CO}_{2}$ ratio. That's three transport equations. That's probably enough. We still want the model to be practical, and we're adding dreaded CPU hours with every new equation, plus uncertainty with every new empirical parameter.

\section{WHAT IS STANDING IN THE WAY OF PROGRESS?}

Miscommunication, in various forms. Fire models serve a fairly small community and have been developed and maintained by literally a few dozen individuals. Many more may have contributed in some way, but relatively few people actually write the programs. There simply aren't enough resources to support more. As a result, those who write the programs find themselves in a peculiar position. They have tremendous influence over the day to day practice of fire protection engineering, but in a strange way are also isolated from it because all of their time is consumed by the never-ending minutiae of making the thing actually work. Few step inside the laboratory except for an occasional tour; most never see real buildings burn except on the evening news. So there is a widening gap between the people who write the programs, the people who run the programs, and the people who are influenced by the results. Everyone involved in the process wants to claim success. That's natural. The modeler is hesitant to express doubts about the increasingly elaborate sub-models demanded by the practitioners who are in fierce competition for projects that inevitably push the model just a bit further than for which it was designed - certainly further than for which it was validated.

$$
\begin{aligned}
\frac{\partial}{\partial t}\left(\bar{\rho} Z_{v}^{2}\right)+\frac{\partial}{\partial x_{j}}\left(\bar{\rho} \tilde{u}_{j} Z_{v}^{2}\right)= & \frac{\partial}{\partial x_{j}}\left(\bar{\rho} D \frac{\partial Z_{v}^{2}}{\partial x_{j}}\right) \\
& -2 \bar{\rho} D \frac{\partial \widetilde{Z}}{\partial x_{j}} \frac{\partial Z}{\partial x_{j}} \\
& +2 \bar{\rho} D \frac{\partial \tilde{Z}}{\partial x_{j}} \frac{\partial \tilde{Z}}{\partial x_{j}} \\
& \left.-\frac{\partial}{\partial x_{j}}\left(\bar{\rho} \widetilde{\left(u_{j} Z^{2}\right.}-\tilde{u}_{j} \tilde{Z}^{2}\right)\right) \\
& +2 \tilde{Z} \frac{\partial}{\partial x_{j}}\left(\bar{\rho}\left(\widetilde{u_{j} Z}-\tilde{u}_{j} \tilde{Z}\right)\right)
\end{aligned}
$$

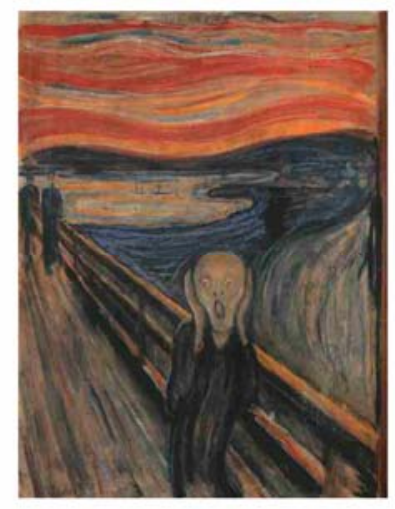

Fig. 3. Browsing through the turbulent combustion literature can be frightening. At left is a transport equation for the sub-grid variance of the mixture fraction. At right, Scream

(1893) by Edvard Munch (Norwegian, 1863-1944), courtesy Nasjonalgalleriet, Oslo.

Perhaps the largest source of miscommunication is turbulence modeling, a subject I touched on above. Turbulence modeling is how we treat the diffusion terms in the governing equations - the viscosity, thermal conductivity and material diffusivity. These terms define dissipative processes at length scales that are far smaller than those that can be directly resolved by the numerical grid in practical simulations. No doubt this is a challenging problem, but progress cannot be made if the practitioners cannot understand what the modelers are trying to say. The techniques are extremely complicated, CPU time consuming, and have less and less connection to the underlying physics that they are 
supposed to be addressing. Worse still, it is often very difficult to determine which methods work, which don't, and which do nothing at all. Graduate students and young researchers, who do the bulk of the programming, often do not or cannot explain why some technique works or doesn't because they are over-shadowed by professors or managers who are disconnected from the numerical details. If the program runs, after months of arduous trial and error, nobody wants to question why. The papers are published, the student is graduated or the researcher promoted, and the numerical technique, for better or worse, enters the literature.

\section{What Can The Model Developers Do To Help?}

If we are to make progress, we all have work to do. Currently, the language and nomenclature used in the turbulent combustion literature is beyond the reach of almost everyone outside of this specialty. I must confess that I do not understand most of the papers I read on the subject, and I have a doctorate in mathematics and have spent the last 14 years writing and applying CFD models! The equation in Fig. 3 was copied from a paper (which I won't cite) that described the implementation of a turbulent combustion algorithm in FDS. While I am gratified to see others working with the model, I am mortified that I cannot understand what they have done. I recognize that the terms on the first line represent the transport of something called the "sub-grid variance of the mixture fraction," but the sources and sinks of this quantity (presumably the rest of the terms on the right hand side), plus the corresponding initial and boundary conditions are a mystery. Rarely are these details discussed, even though the empirical (or to some, completely arbitrary) parameters associated with these terms can number in the dozens. Worse yet, I am worried that the turbulent "statistics" extracted from the resolvable flow variables may be flawed because of known numerical errors in the neighborhood of the flame sheet. These errors are apparent to anyone who has run FDS and noticed slight dips in temperature and species concentrations at the base of a fire plume, known in the numerical community as "undershoots" [10].

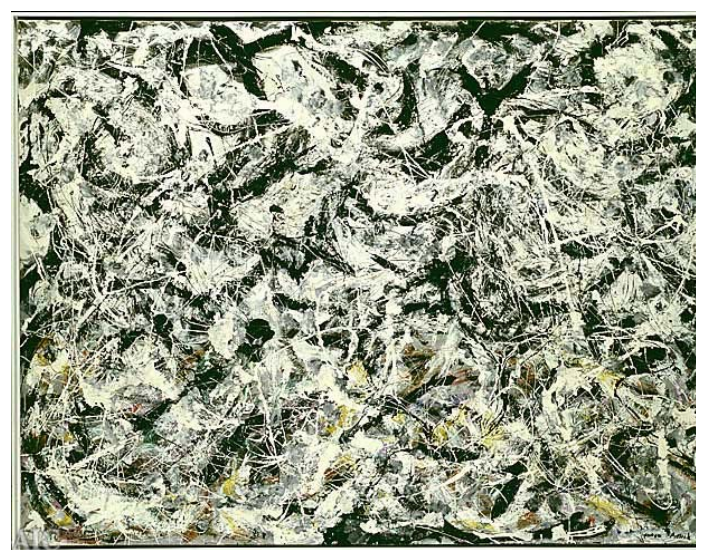

Fig. 4. Greyed Rainbow (1953) by Jackson Pollack (American, 1912-1956). Courtesy The Art Institute of Chicago.

Why does this continue? Probably because even had I reviewed that paper, I would not have been able to offer valid criticism, other than to admit that I did not understand it. There may be a good idea in there somewhere, but who can say? I get a similar feeling when I go to a modern art gallery. Is the painting shown in Fig. 4 the work of a genius? 
Or are we too afraid to say that it is nonsense? The blame should be shared equally between the modelers themselves and the rest of the community, and I'll accept blame as a member of both. We should force turbulence modelers to explain themselves better, and simply referencing another paper is not sufficient. I'll take the first step, as an example, in explaining how we use Large Eddy Simulation in our fire model FDS:

Form an evolution equation for the kinetic energy of the gas by taking the dot product of the momentum equation and the velocity vector $\mathbf{u}$ :

$\rho \frac{D \mathbf{u}}{D t} \cdot \mathbf{u} \equiv \rho \frac{D\left(|\mathbf{u}|^{2} / 2\right)}{D t}=\ldots-\Phi$

and notice a sink term involving the viscous stress tensor $\tau$ and the velocity vector, known as the Dissipation Function:

$\Phi=\tau \cdot \nabla \mathbf{u}=\mu\left[2\left(\frac{\partial u}{\partial x}\right)^{2}+\ldots\right]$

which shows up as a source term in the energy equation:

$\frac{D}{D t}(\rho h)=\ldots+\Phi$

Extra terms have been hidden to make the point more clearly. Nothing has been introduced thus far except the Navier-Stokes equations. This exercise merely explains in mathematical terms how the kinetic energy of the flow is converted into thermal energy by the action of the viscosity, $\mu$ [11]. In the early 1960's, a meteorologist named Smagorinsky [12] suggested, based mainly on dimensional arguments, that the dissipative effect of the viscosity could be represented in a large-scale flow simulation by the expression:

$\mu_{L E S}=\rho\left(C_{s} \Delta\right)^{2}\left[2\left(\frac{\delta \bar{u}}{\delta x}\right)^{2}+\ldots\right]^{1 / 2}$

where $C_{s}$ is an empirical constant, $\Delta$ is the size of a grid cell, and the expression in parentheses has the same functional form as the Dissipation Function. The bar above the velocity component $u$ indicates that this is the resolved value, meaning that it's what's actually being computed, and the $\delta$ 's indicate a finite difference approximation to the partial derivative. The other diffusive parameters, the thermal conductivity and material diffusivity, are related to the modeled viscosity by

$k_{L E S}=\frac{\mu_{L E S} c_{p}}{\operatorname{Pr}} \quad ; \quad D_{L E S}=\frac{\mu_{L E S}}{\rho \mathrm{Sc}}$

The "turbulent" Prandtl number Pr and Schmidt number Sc are assumed to be constant and of order unity, which is more or less true of the actual Prandtl and Schmidt numbers. 
That's it! I have described our implementation of Large Eddy Simulation in a way that anyone with a modest background in fluid mechanics can understand. I started with the basic governing equations to motivate the technique, I explained how these equations have been modified in our numerical model, and I have indicated how many empirical parameters (3) and extra equations ( 0 ) have been introduced. In the documentation for the Fire Dynamics Simulator [13], we go on to discuss the sensitivity of our calculations to these parameters, the accuracy of the predictions, the strengths and weaknesses of the technique, and examples of its use in practical fire simulations. We also make the point, even though it is heresy to the LES purists, that the technique outlined above works largely because it provides us with the minimum amount of "artificial" viscosity to maintain numerical stability in our convective transport algorithm, a lesson learned by Rehm and Baum who for fifteen years employed Eq. (6) successfully without the terms from the Dissipation Function [14]. The adoption of these extra terms in the viscosity model allowed for even less artificial damping because of its tendency to become large only at the onset of a numerical instability. The fact that there was a meaningful physical interpretation of the technique was icing on the cake, and helped gain acceptance by the wider community. Some of these observations, albeit with a different perspective, are echoed in a recent review article on LES [15].

Those reviewing papers, attending conferences, or using CFD models ought to demand this type of explanation. It's not enough for the author or speaker to merely cite someone else, who in turn cites someone else, and so on. Indeed, I cited Smagorinsky's original article above just to be polite - the article is virtually incomprehensible. I cannot even find the simple expression for the modeled viscosity in the paper; I rely instead on review articles by other meteorologists who seem confident that it is there somewhere. God bless them! Confounding Smagorinsky's discussion is the unfamiliar nomenclature and formulations of the governing equations that have been adopted by the meteorological community. We face a similar dilemma, in that turbulence in fire is rarely discussed in the absence of combustion chemistry, hence the name "turbulent combustion." The current literature in this area has become, or maybe has always been, mystifying. Much of the sub-grid phenomena, including combustion, is described in terms of statistical (probability density) functions, like beta-PDF, but with surprisingly little mention of commonly used terms like lower oxygen limit, ignition temperature, and so forth. As with flamelets, it's difficult to know exactly what information is being generated directly from the equations being solved, or even what those equations are. The number of empirical parameters continue to grow, as do the number of new, largely unphysical terms in the equations. To borrow a term invented by the US military to indicate when it has overstepped its original mission, we face in our models equation "creep."

Instead of having each new graduate student add some new term to the expanding equation set, I propose that the students ought to identify terms or routines that have no solid numerical or physical basis, eliminate them, and be rewarded with a degree. The best advice I was given by my advisor in graduate school, an eclectic mathematician named Paul Garabedian, was that any technique or model that could not be described either from memory or with the assistance of a few notes written on one's hand was probably not worth pursuing. The difficulty in cleaning house, numerically speaking, is that someone's favorite term or algorithm is going to have to be dumped unceremoniously at the curb. Graduate students are understandably very hesitant to do this, even though it has to be done to make progress. Computer models often pass from one student to another, each adding some new routine, and no one knows enough about the whole code to surgically remove the fat. A good rule of thumb (known at NIST as 
Baum's Rule) is that the CPU consumption of a given numerical routine ought to be commensurate with the particular phenomenon addressed. For example, the radiation solver in FDS, developed by Simo Hostikka at VTT Finland, consumes about $25 \%$ of the total CPU time. This is reasonable, given that about one-third of the energy transport in a fire is via thermal radiation.

Unfortunately, a trend working against code efficiency is "high performance" computing. In the US and elsewhere, computer scientists at large government labs and elite universities are looking for any viable piece of code to run on "massively parallel architectures." These efforts discourage model developers from making their original codes more efficient. In fact, good "scale-up" is sometimes achieved by sacrificing numerical algorithms that work well on single processors, but less well on multiple processors. Thus, the modelers become dependent on very cumbersome, expensive systems that require constant care and feeding, draining resources from the physical models to support the hardware and software. Often, the objective of the project becomes the parallel computing, rather than the fire modeling. Worse still, these models are almost never used in practice, and they are also of little use in studying the fundamental physics because they rely largely on empirical or ad hoc sub-models.

\section{What Can The Model Users Do To Help?}

Fire modeling has a wider user community outside of academia than other fields of CFD. The benefit of this trend is that the fire models have always been bandied about more, tested, tweaked, cursed. That's how the models have developed as quickly as they have. That's how computers moved from mainframes to "handhelds" in about 20 years. But like computer viruses, there's a price to pay. How do we stop bad fire modeling? Better communication. As I said above, the model developers have to tell the truth about how the models work, and how well they work. But the practitioners have to listen, no matter how dull the discussion about stoichiometry, extinction coefficients, and, yes, turbulence. Too often, model users simply say that they don't have the necessary background to understand the models. That's understandable for the more sophisticated numerical techniques, but not for the physical models. If a paper under review does not explain the model well, the reviewer ought to send it back and get an explanation. Badly written papers should no longer slide through the system because everyone is afraid to admit that they do not understand them.

Another way model users can help improve the models is to exercise new features. I rely a great deal on FDS users who try out new algorithms or routines, usually in ways I never anticipated. This process is often called "beta testing" and it is something all software undergoes in various degrees of formality. With FDS, it is fairly informal. Every modeling effort I take on involves a large amount of routine work, like simple transport from a fire of known size, plus some extra little wrinkle. Modeling wouldn't be fun if not for the wrinkles. I am comfortable applying a new feature if I make it clear to my sponsor that $90 \%$ of what I'm doing is fairly routine and I've got loads of validation work to back it up. But I'll also explain the new idea, what the issues are, that it has never been validated, etc. Usually it's not critical to the outcome, but if it is, I'll just run a couple of simulations to bound the possible range of answers. Validation will come eventually, but in the meantime, we exercise the model because it takes time for new algorithms to shake out. Robust, efficient code evolves. 
Finally, a few words about validation. I am often asked, "Is your model validated?" Rather than answer with a long explanation of verification, validation, model sensitivity, and so on, I simply answer the question with a question: "Is it?" I can point to validation work performed by myself and others, but the most important validation work is that which is done by the user, not the developer. There are several reasons. First, model developers are hesitant to show bad results. Rarely do I read a paper about an inaccurate model, unless it is someone else's model. Second, model developers know how best to run the model to perform well in a given situation. Thus, as a service to both the model developer and to the fire community alike, the user ought to do a modest amount of model validation, both to assure himself that he can properly run the code, and also to assess the accuracy of the model for the given application.

\section{What Can The Rest Of The Fire Community Do To Help?}

Believe it or not, the most influential people in fire modeling are neither the developers nor the users, but rather the project sponsors, journal editors, conference organizers, government managers, fire authorities, and regulators. Rarely do these individuals have a detailed understanding of the computer codes, algorithms, and so on, although many have high levels of education in the various engineering disciplines. The knowledge gap is partly because of age and partly because of time. Modeling is a full-time job. It is difficult to immerse oneself in the thousands of lines of computer code while at the same time take on these other responsibilities. What makes these people important for model development is that they control the two most critical components of a successful program: publishing and funding. Ironically, clarity, simplicity, and practicality in our fire models often work against publication, and can even jeopardize external support. This conclusion is based on my own experience, plus the whisperings of colleagues, that it is difficult to publish so-called "zeroth order" models in the mainstream CFD literature, and that government funding is cut when a numerical model is no longer considered "research" (meaning that it is actually being used). This may sound surprising, but most CFD models that are described in the various journals are rarely used in practice, but they do get published and they do get funded in some way or another. To the university, this constitutes success, and the professor and student are rewarded accordingly. How do we, the fire protection community, reward those who create usable, reliable fire models? At the very least, before recommending a paper for publication or funding a modeling effort, the powers that be ought to ask some hard questions: Is the model being explained clearly? Does the model actually work? Is it practical? Can others use it? Will the model be maintained? The last question is particularly important and is addressed in the next section.

Finally, it's important to look beyond the single individual who oversees the development of a particular model. There are many people who make important contributions but are not rewarded for their efforts. Too often these people are regarded as "programmers" even though writing lines of computer code is a very small part of code development. Regardless of what they are called, they have to understand combustion theory, numerical methods, computer hardware and software, and experimental techniques. It is very difficult to find people with these talents. We need to support them as a community, not just with funding, but also with publishing. Papers on CFD fire modeling should be evaluated in terms of clarity, transparency, and practicality. Just because a paper is incomprehensible does not mean that it must be brilliant work and published. We will not make progress unless the modelers are forced to explain themselves better, and the person best suited for explaining how a model really works is not necessarily the one who's 
name is tagged to it, but rather the one working behind the scenes to ensure that it actually runs.

\section{WHO WILL MAINTAIN THE MODELS?}

Putting aside the various technical debates about LES and RANS, sub-grid chemistry models, differencing schemes and state relations, a subject that rarely surfaces in discussions about fire modeling is: who is going to maintain these enormous computer codes? To most it is a minor consideration; it is just assumed that someone will. However, as the models move from "research" to "practice," we should think more about it, because the improvement of the models is more and more influenced by our ability to keep the codes running as computers change, students graduate, and peoples' minds get soft. The following are some possible answers to the question, "Who will maintain the code?"

The Government: Whose Government? What happened to all those government supported fire labs? Some have been privatized, and even those which haven't still only are partially supported by direct appropriations. Will the privatized labs still give away the models for free? Can they make any money selling the software? CFD fire models have been around since the early 1990's; zone models since the early 1980's. Each group of models are less than a generation old, but already are showing signs of age. At NIST, Walter Jones, the developer of CFAST, a commonly used zone fire model, has retired. Only two people remain who are familiar with the source code. FDS is maintained by myself with some support from over-worked colleagues and several former post-docs, guest researchers and, for lack of a better term, enthusiasts, who are familiar enough with the source code to make meaningful improvements and nurse it into middle age. Smokeview, the visualization companion of FDS, is maintained by only one person, Glenn Forney. The program currently exceeds 50,000 lines of instructions. What will become of it?

Private Companies: Fire models have traditionally been developed at universities and government laboratories and distributed either freely or via some form of consortium or other arrangement. However, there are various successful companies worldwide that have marketed different types of CFD codes, most with a general focus, but some with very specific applications. Some have the ability to model fire and have borrowed techniques that originally developed in research codes at government labs or at universities. So why not leave the care and feeding of fire models to private companies? Several problems: first, the market for fire models is fairly small, and given that earlier versions of these models are already in the public domain, will anyone buy them? Some say that the users will buy the models if more development is done to improve them. But will a private company put money into a program that is marginally profitable?

Academia: This seems like a natural, since students are typically handier with rapidly changing computer technology, they have new ideas, they are energetic, and, most importantly, they're really cheap. The big problem, however, is that students graduate. Can a professor sustain a critical mass of students year after year to maintain a computer code? Who will provide funding for each student? Too often with university research codes, each student adds a subroutine, algorithm, or sub-model; the collective bulk of which over time brings the model to its knees (see above discussion of "equation creep”). Often the various "improvements" are too detailed and scenario-specific to be of much 
use to anyone else, yet both the professor and subsequent students are very hesitant to remove someone else's $\mathrm{PhD}$ thesis from the ever-growing monster.

Professional Societies: Given their very limited resources, professional societies would be the least likely type of organization to take on the task of code maintenance alone. However, they do serve as natural conduits of information, providers of education and training, and professional meetings. These are services that none of the above groups can perform as well, and yet are invaluable in maintaining the free flow of ideas that are necessary to continue model development.

So who will maintain the models? Probably all of the above, either collectively or individually. Given the difficulties faced by each type of organization, collectively is preferable to individually. One way to foster the collective maintenance idea is to make the source codes available. Of course, this is heresy to software vendors, but consider that most scientific software was publicly funded in one way or another. This is certainly true of most of the popular fire models. Making the source codes available means that there will always be a critical mass of people supporting the program and double-checking the algorithms. There still ought to be a single custodian of each model, who may work at any of the above types of organizations. This person would serve as a gatekeeper, making sure that contributed improvements fit within the overall scope of the model. Those familiar with The Adventures of Tom Sawyer (Fig. 5) will recall the scene in which Tom convinces the boys of the town to whitewash the fence for him. The modern analogy is the development of Linux, a computer operating system developed by Linus Torvalds who made the source code freely available to computer enthusiasts worldwide.

As for money, it appears that no one today is getting rich selling fire models. But there clearly is a thriving community of model users who make money selling their expertise. The model developers can support themselves by exploiting their advanced knowledge of the numerics, and hopefully use this income to further the technology.

\section{SUMMARY}

This paper is long on recommendations but very short on concrete steps to move ahead. As CFD modeling of fire becomes more and more routine, more people will become interested in the inner workings of the codes and take the next steps if and only if the algorithms are explained better, the source codes are made available, funding is directed towards methods that work, and publishing clear and concise papers and manuals is encouraged. The very limited set of developers currently maintaining the fire model stockpile cannot move us forward alone. There are very talented individuals throughout the fire community - at universities, at private companies, at the various labs - who are willing to contribute to the effort, and we must foster this cooperative spirit in any way we can.

\section{ACKNOWLEDGEMENTS}

I would like to thank the members of the IAFSS Program Committee for inviting me to present this paper at the Symposium. Thanks also to the following individuals or organizations: the members of the Fire Research Division at NIST who have contributed to the development and application of the Fire Dynamics Simulator, especially to its originators Howard Baum and Ron Rehm, its visualization wizard Glenn Forney, and contributors Ruddy Mell, Kuldeep Prasad, Anthony Hamins, Dan Madrzykowski, Steve Kerber and Doug Walton; our collaborators at VTT Finland, especially Simo Hostikka, 
Timo Korhonen, Jukka Vaari and Jukka Hietaniemi; the financial and organizational support of the US Nuclear Regulatory Commission and the US Bureau of Alcohol, Tobacco and Firearms. Finally, thanks to all those "enthusiasts" at the following organizations who have pitched in to whitewash the fence: ArupFire, Combustion Science and Engineering, FM Global, Hughes Associates, the National Fire Protection Association, Rolf Jensen and Associates, Schirmer Engineering, the Society of Fire Protection Engineers, Tyco Fire Products, Underwriters Laboratories, and the students and professors at Berkeley, Canterbury, Kingston, Maryland, Michigan, Nagoya, Purdue, Seneca College, Victoria, Virginia Tech and Worcester Poly.

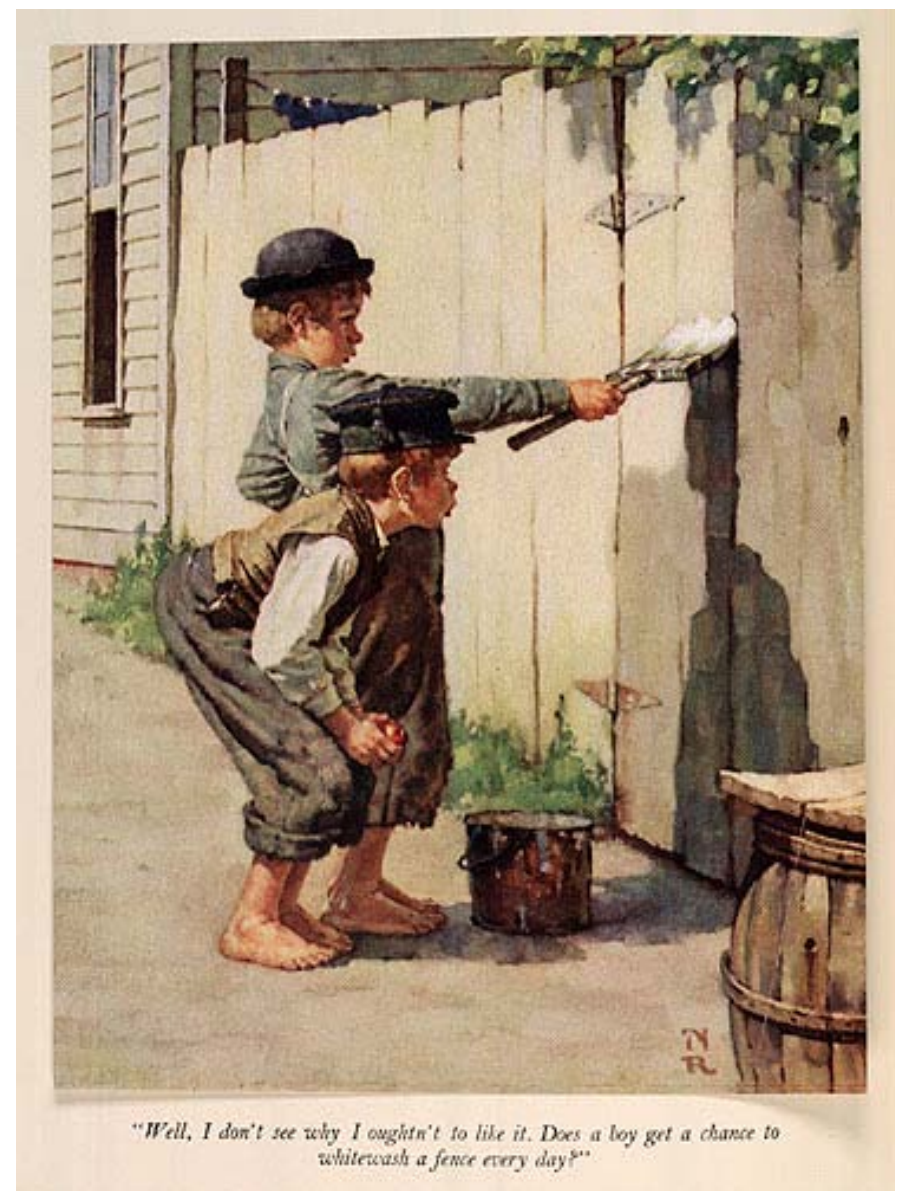

Fig. 5. Illustration by Norman Rockwell for The Adventures of Tom Sawyer (New York: The Heritage Press, 1936). The caption reads, “Well, I don't see why I oughtn't to like it. Does a boy get a chance to whitewash a fence every day?

\section{REFERENCES}

[1] Grosshandler, W.L., Bryner, N., Madrzykowski, D., and Kuntz, K., Report of the Technical Investigation of The Station Nightclub Fire, NIST NCSTAR 2, National Institute of Standards and Technology, Gaithersburg, MD, July 2005. 
[2] Olenick, S. and Carpenter, D., "An Updated International Survey of Computer Models for Fire and Smoke," Journal of Fire Protection Engineering, 13:87110, May 2003.

[3] Di Blasi, C., "Modeling and Simulation of Combustion Processes of Charring and Non-Charring Solid Fuels," Progress in Energy and Combustion Science, 19:71-104, 1993.

[4] Beyler, C.L., "Major Species Production by Solid Fuels in a Two Layer Compartment Fire Environment," in Fire Safety Science - Proceedings of the First International Symposium, pages 431-440, Hemisphere Publishing Company, 1986.

[5] Zukoski, E.E., Toner, S.J., Morehart, J.H., and Kubota, T., "Combustion Processes in Two-Layered Configurations," in Fire Safety Science Proceedings of the Second International Symposium, pages 295-304, Hemisphere Publishing Company, 1989.

[6] Pitts, W.M., "The Global Equivalence Ratio Concept and the Formation Mechanisms of Carbon Monoxide in Enclosure Fires,” Progress in Energy and Combustion Science, 21:197-237, 1995.

[7] Gottuk, D.T. and Lattimer, B.Y., SFPE Handbook of Fire Protection Engineering, chapter Effect of Combustion Conditions on Species Production, National Fire Protection Association, Quincy, MA, 3rd edition, 2002.

[8] Tuovinen, H., Blomqvist, P., and Saric, F., "Modelling of Hydrogen Cyanide Formation in Room Fires,” Fire Safety Journal, 39:737-755, 2004.

[9] Westbrook, C.K. and Dryer, F.L., "Simplified Reaction Mechanisms for the Oxidation of Hydrocarbon Fuels in Flames," Combustion Science and Technology, 27:31-43, 1981.

[10] Oran, E.S. and Boris, J.P., Numerical Simulation of Reactive Flow, Elsevier Science Publishing Company, NY, 1987.

[11] Anderson, D.A., Tannehill, J.C., and Pletcher, R.H., Computational Fluid Mechanics and Heat Transfer, Hemisphere Publishing Corporation, Philadelphia, PA, 1984.

[12] Smagorinsky, J., "General Circulation Experiments with the Primitive Equations I. The Basic Experiment,” Monthly Weather Review, 91(3):99-164, March 1963.

[13] McGrattan, K.B. (editor), Fire Dynamics Simulator (Version 4), Technical Reference Guide, NIST Special Publication 1018, National Institute of Standards and Technology, Gaithersburg, MD, July 2004.

[14] Baum, H.R., Ezekoye, O.A., McGrattan, K.B., and Rehm, R.G., "Mathematical Modeling and Computer Simulation of Fire Phenomenon," Theoretical and Computational Fluid Dynamics, 6:125-139, 1994.

[15] Pope, S.B.“"Ten questions concerning the large-eddy simulation of turbulent flows," New Journal of Physics, 6:1-24, 2004. 